\title{
Cysts of the Optic Nerve Three Cases Associated with Meningioma
}

\author{
A. A. MCNAB* and J. E. WRIGHT† \\ London
}

\begin{abstract}
Summary
Cystic enlargement of the optic nerve sheath is extremely rare. Two cases secondary to a small meningioma in the orbital apex and one secondary to an intraorbital optic nerve sheath meningioma are described, a previously unreported association. High resolution CT scans or MRI can delineate quite small lesions in the orbital apex but a small meningioma within the dural sheath may be missed. Patients with an optic nerve cyst without an apparent cause should therefore be scanned over a number of years to exclude the presence of a meningioma.
\end{abstract}

In reviewing the records of all patients presenting to the Orbital Clinic at Moorfields Eye Hospital between 1968 and 1986, three cases of cystic expansions of the sheath of the intraorbital optic nerve were found amongst almost 3,000 patients. Two of these cases were mentioned in a list of orbital tumours compiled by Lloyd, ${ }^{1}$ but their association with meningioma was unknown at that time, and the diagnosis was made only subsequently. One further case has since presented which was also secondary to meningioma.

Optic nerve cysts are rare but have been found to accompany a variety of pathological entities. A small number had no associated lesion and probably represented a primary disorder. No cases have been reported in association with meningioma.

\section{Case Reports \\ Case 1}

A 53-year-old male presented in November 1977 complaining that for a year he had experienced dimming of his right vision for half an hour on waking. For ten months he had noticed horizontal diplopia on right lateral gaze. He was examined elsewhere and found to have right optic disc swelling with a normal left disc. Investigations including lumbar puncture, syphilis serology, skull x-ray and radionucleide brain scan were normal. An orbital venogram however showed failure of filling of the third part of the superior ophthalmic vein suggesting compression in the posterior third of the orbit. He was referred to the Orbital Clinic at Moorfields Eye Hospital.

In the six weeks prior to being seen at Moorfields he had also noticed episodes of transient blurring of the right vision during the day, lasting several minutes each time, in addition to the same symptom on waking. Corrected visual acuity was $6 / 6$ right and $6 / 5$ left. There was a subtle relative right afferent pupil defect and Goldmann perimetry revealed an enlarged blind spot, some upper nasal constriction and a nasal step. Ocular movements were slightly restricted in abduction but more markedly so in elevation. There was $2 \mathrm{~mm}$ of right axial proptosis. On ophthalmoloscopy there was right disc swelling but no opticociliary shunt vessels or choroidal folds. The right ocular examination and the rest of the neurological and general examinations were

*Fellow, Orbital Clinic, Moorfields Eye Hospital, London.

†Consultant, Orbital Clinic, Moorfields Eye Hospital, London.

Correspondence to: Mr J. Wright, Moorfields Eye Hospital, City Road, London EC1V 2PD.

*Work supported by the Royal Australian College of Ophthalmologists-OPSM Travelling Fellowship. 
normal. B-scan ultrasonography demonstrated a large mass immediately behind the globe which could not be separated from the optic nerve.

A right lateral orbitotomy was performed and a markedly enlarged optic nerve sheath identified. The sheath was incised and clear colourless fluid under considerable pressure was released. The remainder of the optic nerve and orbit appeared normal. The nerve sheath was biopsied and showed normal dura only.

Post-operative acuity was $6 / 9$ right. There was a dilated unreactive pupil in addition to a subtle relative afferent pupil defect with normal extraocular movements. The pupil returned to normal size and reactivity in several weeks apart from the persistent mild afferent defect. The disc swelling settled and proptosis resolved with no difference between the two sides. Perimetry showed a normal blind spot, no constriction nasally, but a residual nasal step.

The patient's visual obscurations also resolved but the diplopia persisted. These symptoms and the clinical signs remained stable for three years and then the patient again complained of dimming of vision, without fluctuation. Acuity was now 6/9 right and 6/5 left, there was a right relative afferent pupil defect and optic atrophy, again without shunt vessels. There was a right axial proptosis of $2 \mathrm{~mm}$.

A skull $x$-ray now showed hyperostosis of the inner aspect of the sphenoid wing adjacent to the optic foramen, and CT scanning showed thickening of the apical optic nerve with positive contrast enhancement. There was also contrast enhancement in the right cavernous sinus (Fig.1). A carotid angiogram revealed a tumour blush in the orbital apex and irregular constriction of the carotid siphon within the cavernous sinus.

At craniotomy the clinical diagnosis of men-

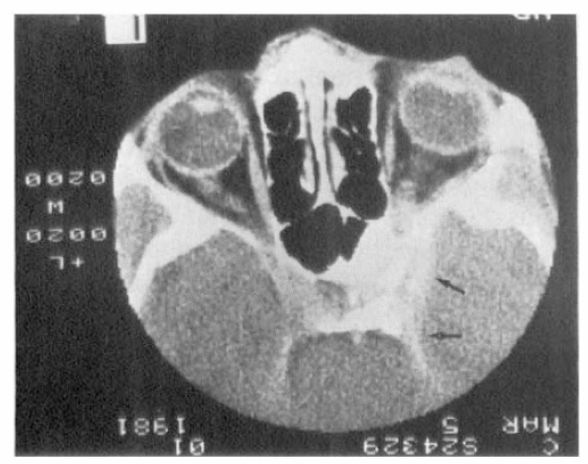

Fig. 1 Case 1. Axial CT scan with intravenous contrast shows thickening of the apical optic nerve with associated bony sclerosis and contrast enhancement in the region of the cavernous sinus (arrows). Biopsy confirmed the diagnosis of meningioma. ingioma was confirmed, the tumour was debulked and the optic canal deroofed. Vision remained at $6 / 9$ but with improved field and colour vision. Six months after surgery, visual acuity had dropped to $6 / 18$. The patient was subsequently lost to followup.

\section{Case 2}

This woman was first seen by her local ophthalmologist in 1969 at the age of 35 complaining of blurred left vision over a period of several months. Acuities were recorded at $6 / 6$ right and 6/9 left and some temporal disc pallor noted on the left. A clinical diagnosis of optic neuritis was made. However within a year the left vision had gradually and painlessly deteriorated to $1 / 60$ in the left eye with gross optic atrophy. Skull $x$-rays with foramen views and syphilis serology were negative and no further action was taken. Three-and-a-half years later proptosis was first noticed. Plain films were again normal but the patient was referred to the Orbital Clinic with a suspected diagnosis of optic nerve sheath meningioma.

When first seen in the clinic in March 1974, the right visual acuity was $6 / 6$ with a normal field. The left visual acuity was hand movements only with a marked relative afferent pupil defect and optic atrophy without shunt vessels. There was $5 \mathrm{~mm}$ of proptosis with $3 \mathrm{~mm}$ of lateral displacement of the left globe. Ocular movements were moderately restricted in all directions. Neurological and general examinations were normal. Orbital venography showed obstruction of the third part of the superior ophthalmic vein and B-scan ultrasonography revealed enlargement of the optic nerve immediately behind the globe.

Lateral orbitotomy was performed and the optic nerve identified. It was enlarged to $8 \mathrm{~mm}$ in diameter with a bluish distended sheath. The sheath was incised and clear fluid under pressure was released. The underlying nerve appeared atrophic. The sheath was stripped from the nerve over a distance of $10 \mathrm{~mm}$ and submitted for pathological examination. The remainder of the orbit appeared normal. The vision post-operatively was bare perception of light. Microscopic examination of the excised nerve sheath revealed normal dura with underlying normal arachnoid cells.

The relative left proptosis was reduced from $5 \mathrm{~mm}$ to $2 \mathrm{~mm}$ after post-operative oedema settled. However, a year and a half after the surgery, the proptosis increased to $6 \mathrm{~mm}$ within the space of six months. There was no perception of light. A CT scan (matrix $320 \times 320$ ) was performed and showed a mass in the orbital apex. It was decided to re-explore the orbit through a lateral orbitotomy. Abnormal dense tissue with a fibrous texture was 
found extradurally in the posterior third of the orbit and biopsied. Histopathological examination now revealed meningioma with prominent fibrosis. On this basis a further operation was performed with removal of the globe and optic nerve and clearance of the orbital apex.

The patient was last seen in August 1981, five years after her last operation, with no evidence of recurrence, but has since been lost to follow-up.

\section{Case 3}

A 61-year-old woman had first been aware that her right eye was weak at the age of thirty, but had not sought medical attention. Two weeks before being seen in the Orbital Clinic in October 1975, she had noticed a sudden deterioration in her right vision. There were no other symptoms.

Visual acuity was counting fingers at one metre on the right and $6 / 9$ left. There was a right relative afferent pupil defect and $6 \mathrm{~mm}$ of axial proptosis. Ophthalmoscopy revealed optic atrophy with choroidal folds at the posterior pole, but no shunt vessels.

Plain $\mathrm{x}$-rays were normal but conventional tomography suggested an intraconal mass, a finding substantiated on orbital venography by elevation and stretching of the superior ophthalmic vein. Ultrasonography also revealed a large mass commencing a few millimetres behind the globe and inseparable from the optic nerve. A CT scan (matrix $160 \times 160$ ) was performed and showed an intraconal mass directly behind the globe.

A lateral orbitotomy was performed. A large smooth-walled cystic mass surrounded the optic nerve anteriorly. Within the distended sheath was a highly vascular mass based infero-laterally. The optic nerve sheath distension extended towards the orbital apex. On incising the sheath, clear fluid was obtained. The optic nerve with the tumour within

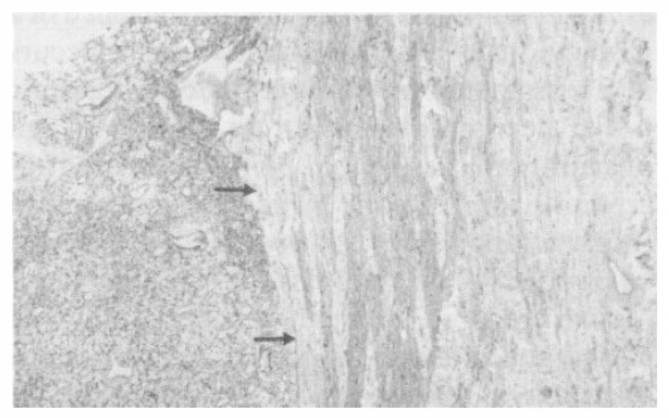

Fig. 2 Case 3. Angioblastic meningioma abutting against the optic nerve (arrows), cut longitudinally (to the right). Haematoxylin and Eosin, $x$ 47.5. its sheath was transected at the globe and the orbital apex and removed.

Histopathological examination revealed a lesion with the features of an angioblastic meningioma, showing areas of xanthomatous degeneration. A mass composed largely of thin walled vascular channels with intervening foci of cells with foamy cytoplasm was situated beneath the dura and abutted against the nerve (Figs. 2 and 3 ). In eleven years of follow-up there has been no evidence of recurrence and the blind eye has remained comfortable with a well perfused anterior segment.

\section{Discussion}

There are few case reports of cystic lesions of the optic nerve involving intraorbital optic nerve sheaths. Generalised distension of the optic nerve sheaths by cerebrospinal fluid probably occurs in many patients with raised intracranial pressure. It may be the only abnormal feature on $\mathrm{CT}$ in a patient with benign intracranial hypertension and papilloedema. ${ }^{2}$ The expansion is not a true cyst of the optic nerve but a passive response of the optic nerve sheaths to prolonged raised pressure.

Cysts involving other parts of the optic nerve have been recorded. Cysts of the optic nerve head have been found as congenital anomalies $^{3.4}$ and a cysticercus cyst has been observed at the same site. ${ }^{5}$ Two cases of arachnoid cyst affecting the intracranial optic nerve have been reported ${ }^{6}$ in addition to a cyst lined by stratified squamous epithelium, thought to represent a craniopharyngioma. ${ }^{7}$ Gliomas of the optic nerve and chiasm may be cystic also. ${ }^{8}$ An intracranial arachnoid cyst situated in the anterior middle cranial fossa, a common site for such a lesion, has been found to expand into the orbit along the optic canal in one case, and to be associated with raised intraocular pressure and an arcuate field defect. ${ }^{9}$

Thirteen cases of cystic lesions of the optic nerve confined to the orbit have been reported..$^{10-21}$ Only one was probably associated with meningioma. There are only four cases of discrete optic nerve cysts among those reported where no associated pathology was found and each cyst appeared to have arisen de novo. ${ }^{10-13}$ These may represent a type of arachnoid cyst confined to the optic nerve. The remainder had a variety of associated lesions: optic nerve glioma, ${ }^{14}$ von Reck- 


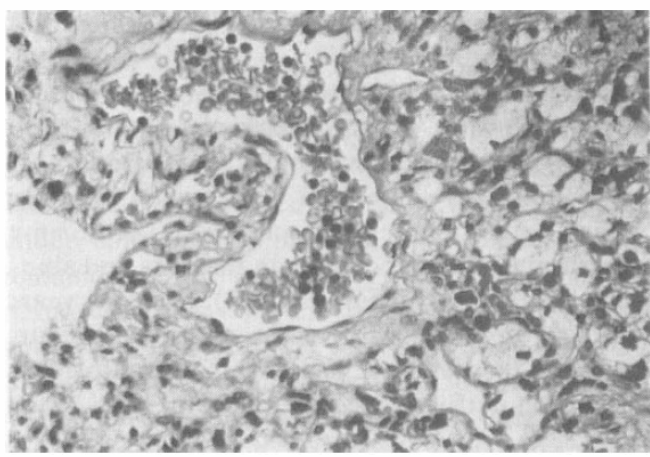

Fig. 3 Case 3. Section of angioblastic meningioma showing thin walled vascular channels and intervening foci of cells with foamy cytoplasm representing xanthomatous degeneration. Haematoxylin and Eosin, $x 560$.

linghausen's disease,,$^{15}$ fronto-temporal porencephalic cyst,${ }^{10}$ haemangioma of the optic nerve sheath, ${ }^{16,17}$ and following closed trauma. ${ }^{18}$ One of the two cases of "arachnoid cyst" of the optic nerve reported by Miller and Green ${ }^{10}$ was an incidental finding in a 56-yearold woman with a fronto-temporal porencephalic cyst decompressed by a cysto-atrial shunt. The patient died from haemorrhage into the porencephalic cyst and a small cystic expansion of the optic nerve sheath was found post-mortem. It was the authors' belief that the "cyst" found in this case probably represented a localised expansion of the optic nerve sheath due to transmission of raised intracranial pressure. It probably played no part in the pathogenesis of the optic atrophy which was bilateral.

Bane ${ }^{19}$ described a patient with a cyst of the dural sheath, the histological features of which were highly suggestive of meningioma but this diagnosis was not postulated. Two patients were thought to have optic nerve meningioma prior to surgery, ${ }^{10.16}$ but no such tumour was found. The three patients described in this paper represent a previously unrecorded association between a cystic enlargement of the optic nerve sheaths and a meningioma in the apex of the orbit (cases 1 and 2) or in the intraorbital optic nerve sheaths (case 3 ).

Many tumours occupy the apex of the orbit without causing distension of the optic nerve sheath distal to the lesion. The lesions described in cases one and two occupied the subdural space and their relation to the flow of cerebrospinal fluid (CSF) may be the most important factor in producing an accumulation of fluid within the optic nerve sheath. Meningiomatous tissue could act in a ballvalve manner, only allowing CSF to pass in one direction into the intra-orbital subarachnoid space. Such a mechanism is unlikely for it would need an increase in the central CSF pressure to overcome the ballvalve as the pressure and size of the cyst increased.

Blood vessels within the tumour may be abnormally permeable and act as a source of fluid. The tumour in our third case was highly vascular as was the haemangioma described by Harris et al. ${ }^{17}$ and both may have accumulated fluid in this way. A haemorrhage into an isolated cavity can continue to draw in fluid as high molecular weight blood breakdown products are unable to escape. Cystic hygromas following subdural haematomata are thought to arise in this manner, and this is the probable mechanism in the case reported by Hupp et al. ${ }^{18}$ which followed closed head trauma.

CSF is normally produced by the choroid plexus of the ventricles. However, as much as $50 \%$ may be produced by the brain itself. ${ }^{22}$ If tissues of the optic nerve (essentially brain white matter) were capable of CSF production it might explain the source of the fluid in cases with obstruction to drainage of CSF into the cranial subarachnoid space from the orbit.

Whatever the source of the fluid, it can interfere with optic nerve function. In Case 1, there was a definite improvement in vision after decompression, despite the presence of a meningioma. The same improvement occurs in a proportion of patients undergoing optic nerve decompression for disc swelling and obscurations associated with benign intracranial hypertension. ${ }^{23}$ The papilloedema in the case reported by Smith et al. ${ }^{20}$ was relieved by optic nerve decompression. Another case ${ }^{18}$ had an enlarged optic nerve sheath following closed head trauma associated with a venous obstructive retinopathy. Decompression of the ballooned nerve sheath led to visual improvement and a decrease in the size of the sheath. In several cases decompression of the 
distended nerve sheath failed to improve vision $^{12,21}$ although the disc swelling diminished in Saari's case.

Our Case 1 had an improvement in vision after decompression despite the presumed presence of the meningioma. As noted above, others have reported visual improvement after optic nerve decompression for cystic expansion of the dural sheath. We believe that an optic nerve cyst which is interfering with the function of the nerve should be decompressed by removing a small portion of the nerve sheath to release CSF.

Arachnoid cysts of the optic nerve arising without any associated pathology are extremely rare. The clinician who detects a localised distension of the optic nerve sheath with CT or NMR scanning, or at surgery, should search for a causative lesion within the orbit. The present cases were seen before the advent of high resolution CT and MRI. Modern imaging systems should detect quite small abnormalities in the apex of the orbit and avoid the delay in the diagnosis which occurred in two of our patients. If no cause is found for the cyst then the patient should be reassessed for several years before concluding that the lesion was a simple arachnoid cyst.

\section{References}

${ }^{1}$ Lloyd GAS and Ambrose JAE: An evaluation of CAT in the diagnosis of orbital space-occupying lesions. In du Boulay GH, Moseley IF eds. First European Seminar on Computerised Axial Tomography in Clinical Practice, Berlin, Springer, 1977, 15460.

${ }^{2}$ Moseley IF and Sanders MD: Optic nerve disease. In Computerised Tomography in Neuro-Ophthalmology, London, Chapman and Hall, 1982, 154-60

${ }^{3}$ Agatson SA: Congenital cyst of the optic nerve. Am J Opthalmol 1944, 27: 278-81.

${ }^{4}$ Wright P: Meningocoele of the optic disc. $\mathrm{Br} \mathrm{J}$ Ophthalmol 1960, 44: 570-1.

${ }^{5}$ Bawa YS and Wahi PL: Cysticercosis cellulosae of the optic disc with generalised cysticercosis. $\mathrm{Br} \mathrm{J}$ Ophthalmol 1962, 46: 753-5.

${ }^{6} \mathrm{Holt} \mathrm{H}$ : Cysts of the intracranial portion of the optic nerve. Am J Ophthalmol 1966, 61: 1166-70.

${ }^{7}$ Walsh FB, Chambers JW, Lloyd LA: The ocular signs of tumours involving the anterior visual pathways. Am J Ophthalmol 1956, 42: 347-7.

${ }^{8}$ Brihaye M, Graff G, Brihaye $\mathrm{J}$ et al.: Voluminous cystic glioma of the chiasm with predominant cerebral symptomatology. Acta Neurol Psychiatr Belg 1961, 61: 525-38.

${ }^{9}$ Krohel GB and Hepler RS: Arachnoid cyst invading the orbit. Arch Ophthalmol 1979, 97: 2342-4.

${ }^{10}$ Miller NR and Green WR: Arachnoid cysts involving a portion of the intraorbital optic nerve. Arch Ophthalmol 1975, 93: 1117-21.

${ }^{11}$ Brihaye M: Cysts of the optic nerve. Presented at the European Ophthalmic Pathology Society (jointly held with Verhoeff Soc), Washington, Apr 1976.

12 Saari M, Mustonen E, Palva A et al.: Arachnoid cyst of the intraorbital portion of the optic nerve with unilateral disc oedema and transient shallowing of the anterior chamber. Acta Ophthalmol 1977, 55: 959-64

${ }^{13}$ Daniels DL, Davis JP, Kay MC et al.: Opacification of an optic nerve sheath cystic tumour with intrathecally injected metrizamide. AJNR 1986, 7: 976-7.

${ }^{14}$ Wolter JR and McKenney MJ: Collateral hyperplasia and cyst formation of orbital leptomeninx secondary to optic nerve glioma. Am J Ophthalmol 1964, 57: 1037-42.

${ }^{15}$ Spencer WH: Primary neoplasms of the optic nerve and its sheaths: clinical features and current concepts of pathogenetic mechanisms. $\mathrm{Tr} \mathrm{Am}$ Ophthalmol Soc 1972, 70: 490-528.

${ }^{16}$ Schneider R: Angioretikulom des Sehnerven. Albrecht von Graefe's Arch Klin Ophthalmol 1942, 145: 163-78.

${ }^{17}$ Harris GJ, Sacks JG, Weinberg PE et al.: Cyst of the intraorbital optic nerve sheaths. $A m$ J Ophthalmol 1976, 81: 656-60.

${ }^{18}$ Hupp SL, Buckley EG, Byrne SF et al.: Post-traumatic venous obstructive retinopathy associated with enlarged optic nerve sheath. Arch Ophthalmol 1984, 102: 2546.

${ }^{19}$ Bane WC: Cyst of dural sheath of optic nerve. Am J Ophthalmol 1920, 1: 17-20.

${ }^{20}$ Smith JL, Hoyt WF, Newton TH: Optic nerve sheath decompression for relief of chronic monocular choked disc. Am J Ophthalmol 1969, 68: 633-9.

${ }^{21}$ Wojno T, Beck RW, Grosserode R: Bilateral optic nerve sheath enlargement. Ophthal Surg 1986, 17: 584-9.

${ }^{22}$ Black PM and Conner ES: Chronic raised intracranial pressure. In Asbury AK, McKhann GM, McDonald WI eds. Diseases of the Nervous System. Philadelphia, WB Saunders, 1986, 1054-5.

${ }^{23}$ Galbraith JEK and Sullivan JH: Decompression of the perioptic meninges for relief of papilloedema. Am J Ophthalmol 1973, 76: 687-92. 